\title{
A user-centric economic-driven paradigm for rate allocation in non-orthogonal multiple access wireless systems
}

\author{
Panagiotis Vamvakas ${ }^{1}$, Eirini Eleni Tsiropoulou ${ }^{2}$ and Symeon Papavassiliou ${ }^{1 *}$ (D)
}

\begin{abstract}
In this paper, a novel approach towards optimizing users' rate allocation and price customization in a nonorthogonal multiple access (NOMA) wireless network under quality of service (QoS)-differentiated requested services is proposed and studied. A multi-service wireless system is considered, where each user's QoS requirements are reflected through a utility function, alongside his willingness to pay for the corresponding service. Within this setting, in order to jointly allocate the customized price and rate, a two-variable optimization problem arises. Based on the principles of S-modular theory, the above two-variable (rate and price) optimization problem is modeled and solved as a distributed non-cooperative game. The existence and convergence to the Nash equilibrium point with reference to both user's uplink transmission rate and price is proven. The proposed approach, allowing for decision-making at the user side, is well aligned with the self-optimization and self-adaptation objectives of future emerging 5G wireless networks. The performance evaluation of the devised framework is conducted via modeling and simulation under various scenarios, and the numerical results clearly demonstrate its superiority against other existing approaches.
\end{abstract}

Keywords: Customized pricing, Rate allocation, Heterogeneous services, User-centric management, Non-orthogonal multiple access, S-modular theory

\section{Introduction}

Next-generation communication technologies, the diversification of mobile services, and the users' demand for higher quality of service (QoS) performance have posed quite stringent and demanding challenges to wireless Internet service providers (WISPs). 5G wireless networks require highly spectral-efficient multiple access techniques that play a significant role in optimizing the resource allocation process and improving the performance of the mobile communication system. Given the physical constraints associated with radio resource management, quite often high importance and priority is given at reducing interference and/or power consumption, towards improving energy efficiency. This happens however at the cost/trade-off of achieving lower data rates for the users, depriving them from fully exploiting system's capabilities. Non-orthogonal

\footnotetext{
* Correspondence: papavass@mail.ntua.gr

${ }^{1}$ School of Electrical \& Computer Engineering, National Technical University of Athens, Zografou, 15773 Athens, Greece

Full list of author information is available at the end of the article
}

multiple access (NOMA) technique has been acknowledged as an effective technique for providing high data rates in $5 \mathrm{G}$ systems, especially in the uplink communication [1]. Nevertheless, in order to further improve user satisfaction from the provided services, while getting aligned with the vision of subscriber self-optimization and self-adaptation in future wireless networks, sophisticated user-centric resource management approaches are required.

\subsection{Related work}

The problem of efficient resource management in wireless networks has been broadly addressed in the literature, especially via utilizing game theoretic approaches, which support the autonomous behavior of the mobile user [2]. Power control problems have been extensively studied mainly from the aspect of reducing battery drain and enhancing energy efficiency [3-5]. By adding more degrees of freedom alongside power control, data rate allocation becomes feasible with the authors in [6-8] to convert a two-variable problem to a single parameter optimization 
expressed as the ratio of rate to the respective power. Following the same paradigm, the authors in [9] propose a spectrum allocation framework in cognitive radios following the load balancing concept in distributed computer systems. Furthermore, in [10], the joint allocation of power and rate is examined as an actual two variable non-cooperative game via centrally imposed usage-based pricing, while in [11], a similar approach is extended to two-tier femtocell architectures. Also, centralized approaches targeting at maximizing the secrecy sum rate in a NOMA system have been proposed [12]. Although substantial improvements are achieved, in these works, rate allocation is suppressed due to the priority of minimizing power consumption $[13,14]$.

Considering the class of problems concentrating mainly on rate allocation, in [15], a constrained throughput maximization problem is examined including stochastic control parameters towards safeguarding fairness conditions among the users. In [16], a sum rate optimization problem for multiple-input multiple-output (MIMO) NOMA systems is studied with constraints considering the total transmission power and the minimum rate.

At the same time, several attempts have been promoted to studying the problem of resources' pricing. In $[17,18]$, the nature of prices is fixed, with the users being charged a flat rate or different classes of static prices are applied to users based on their traffic requirements, respectively. The alternative of day-ahead pricing is examined in [19], with the WISP announcing the time-dependent prices 1 day in advance. In [20], Zheng et al. study a secondary data market, in which users can buy and sell left over data caps from one another. The authors derive users' optimal behavior and propose an algorithm for the Internet service providers (ISPs) to match the buyers and the sellers. Considering a similar trading model, in [21], users' decisions about their usage at different times of the billing cycle are discussed. In [22], the authors address mobile users' data usage behavior, through the study of network effects as they emerge by the joint consideration of elements both from social domain (e.g., relationships) and the physical domain (e.g., congestion). The joint pricing and data usage management problem is formulated as a Stackelberg game, where the WISP acts as leader announcing the price to the users, who follow by determining their data usage. In $[23,24]$, a comprehensive literature review on applications of economic and pricing models for resource management in cloud networking and the Internet of Things, respectively, is provided.

The majority of all the relevant works regarding the radio resource management have concentrated on power control and/or sum rate maximization via centrally imposed pricing for interference mitigation, thus failing to provide satisfactory data rates for each individual user and to recognize the high variability among the users' service requirements. Furthermore, even though that the resource management based on game theoretic approaches is a very well-established research field in the literature, very few research works deal with the combined problem of resource management and price customization, especially when the decision-making is performed at the user side in a distributed manner.

\subsection{Contributions}

The main contributions of this article lie in the area of enabling users to actively correlate resource allocation, QoS differentiation, and price customization, towards improving their overall satisfaction under a user-centric paradigm. To the best of our knowledge, this is the first time that an intelligent mobile user is able to determine in a distributed manner his optimal rate and the corresponding price that he is willing to pay in order to achieve it. The proposed rate control and customized pricing framework has been developed aiming 5G multi-service non-orthogonal multiple access wireless networks. It should be clarified that the proposed framework is not just another work in the field of resource management which adopts game theory, but provides a novel framework dealing simultaneously with rate control and customized pricing from an autonomous user behavior point of view, in a $5 \mathrm{G}$ multi-service NOMA wireless environment.

Specifically, in this paper, a different philosophy is followed where the users, depending on prespecified/targeted signal-to-interference-plus-noise ratio (SINR) values, as they are indicated by their requested service, prioritize the allocation of the available bandwidth among them in a real-time dynamic manner by disclosing to the WISP how much price they would accept to be charged in order to fulfill their QoS prerequisites. Hence, both variables (i.e., rate and price) are jointly determined not only in an optimal and real-time manner but also in a distributed and user-centric attitude aiming at the maximization of the users' net utilities, reflecting the overall improvement of the users' satisfaction regarding their service experience. Considering that the intelligence is provided by the mobile node, the proposed framework aims at the optimization of the naturally emerging trade-off between QoS prerequisites satisfaction and cost of resources, with reducing any interaction with the WISP. The above innovation is well aligned with networks providing heterogeneous services as in $5 \mathrm{G}$ systems, where each user or group of users may have considerably different demands. It is noted that in such an environment a centrally determined pricing solution would disregard user segmentation and could damage fairness and proper resource usage instead of improving it. 
Following this consideration and motivation, and in order to jointly allocate the customized price and rate, an optimization problem of two variables arises, which is modeled and solved as a distributed non-cooperative game. The corresponding problem is tackled by optimizing each user's net utility function and capitalizing on the principles of multi-variable S-modular theory. In the following, we refer to this problem (and corresponding game) as joint non-cooperative utility-based uplink transmission rate and customized price control (RP) problem. The concluding point of the two-variable RP game is a Nash equilibrium (NE) point that jointly determines the optimal price and users' rates, in the sense that there is no user that would opt to deviate from this point, since his perceived satisfaction cannot be further enhanced by making any change to his strategy individually. It should be also highlighted that in the majority of the literature, which deals with resource management based on S-modular theory, single-variable optimization problems have been primarily considered [25-27]. In this paper, the S-modular theory is applied to a multi-variable optimization problem, i.e., transmission rate and customized price control, while the extension and proper adaptation of the theory from single-variable to multi-variable optimization problems is not trivial [26].

In contrast to [4], where the uplink transmission power control problem was treated while considering customized pricing, in this work the nature of resource is different, i.e., uplink transmission data rate, which results in completely different users' competition behavior pattern. Specifically, in [4], a higher price is concluded for a specific user as a consequence of an increase in his uplink transmission power. This behavior incentivizes the rest of the group to intelligently choose their uplink power towards reaching a price reduction. This type of users' behavior was captured in [4] by the submodular games. These types of games are associated with the strategic substitutes, meaning that if one player increases his action, this would stimulate the other players to follow an opposite action. In contrast, in the current research work where the users have control over their transmission data rate, a higher data rate claim from some users concludes to higher prices for themselves. Thus, the rest of the users in order to withstand bandwidth competition also accept to be charged with higher prices, so as to maintain their achieved data rates. Therefore, if some users agree to pay more in order to increase their data rates, the other competing users follow the same strategy. This competition behavior pattern is captured through the supermodular games, where the actions of one player lead to similar actions from the other players and not to the opposite actions, as in submodular games adopted in [4].
The performance evaluation of the proposed approach is conducted via modeling and simulation under different pricing alternatives and various scenarios with respect to the number of users. Extensive numerical results indicate the superior performance of the proposed approach when compared to alternative state of the art resource allocation approaches, in terms of achieved data rate and energy efficiency. Our results further confirm that the energy efficiency that can be achieved as outcome of the resource allocation process is significantly enhanced by increasing the degrees of freedom the user is offered. For practical and implementation mainly purposes, we also evaluated and compared several alternatives to show the realistic application of the proposed framework, which essentially targets at the computational complexity reduction. Such alternatives mainly reflect a blend of business and technical differentiation characteristics through the introduction of different user partitioning strategies mainly from the pricing point of view.

\subsection{Outline}

The organization of the remaining of the paper is as follows. Section 2 presents the system model and the associated proposed net utility functions. In Section 3, we provide a fundamental introduction to S-modular games and their main properties, while in Section 4 the combined optimization problem is formally described and addressed as a two-variable S-modular game. In Section 5 , the convergence of the RP game to the NE point with reference to both variables (i.e., price and data rate) is studied, and a distributed and iterative algorithm that identifies this point is introduced. In Section 6, a comparative performance evaluation is presented illustrating the strategic and practical advantages of this approach against other existing works, while finally Section 7 presents some concluding remarks.

\section{System model and utility functions}

\subsection{System model}

In the following, the uplink of a NOMA-based wireless network, including disparate user types exchanging data under the system's physical limitations, is considered. To preserve simplicity in the presentation without harming the validity of our analysis and results, in this paper, the notion of time slot $t$ is omitted.

We assume $|N|$ continuously backlogged users placed around a base station (BS) with coverage radius $\mathcal{R}$, requesting elastic or inelastic services, where $N$ denotes their corresponding set. Key characteristics of the elastic services include high throughput demand and high delay tolerance [25]. However, from a QoS satisfaction perspective, a distinctive feature of the elastic services is that they are shiftable in time, and as a consequence, the corresponding users can properly reshape and adjust 
their demand by accounting for the respective price. On the contrary, the inelastic services are constrained by "hard" short-term data demand that needs to be achieved within a specific time frame. In turn, these requirements are translated into a specific user behavior, where the user is reluctant to consume the service when QoS drops below a pre-determined threshold.

Given the physical, technical, and economical constraints associated with both the users and the WISP, the involved resources, i.e., power, rate, and price are upper bounded $P_{i}^{M i n} \leq P_{i} \leq P_{i}^{M a x}, r_{i}^{M i n} \leq r_{i} \leq r_{i}^{M a x}$, and $c_{i}^{M i n} \leq c_{i} \leq$ $c_{i}^{\text {Max }}$, with the maximum feasible rate values being differentiated by each service type. In this work, we consider the price to be a dimensionless unit adjustable by the users; however, it can be translated into actual monetary units, thus reflecting the realistic economical dimension of this approach. The channel gain between the $i$ th user and the base station is denoted by $G_{i}$, and $G_{i}=\frac{K_{i}}{d_{i}^{i}}$, where $K_{i}$ denotes the Rayleigh fading channel gain, $a$ is the path loss factor and $d_{i}$ denotes the distance from the $i$ th user to the base station.

Employing the NOMA scheme in the uplink communication of a wireless network, its basic characteristic is that the users with improved channel gain sense the interference caused by the users with worse channel conditions, while the reverse does not hold true due to the successive interference cancelation (SIC) technique. Without loss of generality, the channel gains are sorted as $G_{1} \leq \ldots \leq G_{|N|}$. Following the NOMA protocol, SIC is performed at the users. Consequently, the $n^{\text {th }}$ user detects the signal from the $i$ th user, where $n<i$ and then removes the included message from his observation in a successive manner. The signal from the $n^{\text {th }}$ user, $i>n$, is considered as noise at the $i$ th user. Thus, it is observed that the users with worse channel gain can exclude the signals stemming from users with better channel gain in a successive manner (i.e., SIC technique) from their sensed interference. As a result, each user defines his transmission characteristics through his SINR, noted as:

$$
\gamma_{i}=\frac{W}{r_{i}} \frac{G_{i} P_{i}}{\sum_{j=i+1}^{|N|} G_{j} P_{j}+I_{0}}
$$

where $W[\mathrm{~Hz}]$ represents the spread spectrum bandwidth, $G_{i}$ is the channel gain from user $i$ to the BS, $I_{O}$ $[\mathrm{W}]$ is the Additive White Gaussian Noise, and $P_{j}$ refers to the power of each user $j, j>i+1$, [25]. The formulation of the SINR follows the corresponding formulation in the seminal paper [25]. As it will be explained in detail in Section 4, the two-variables that are determined by the user are the data rate $r_{i}$ and his willingness to pay for the received service, which is expressed through the price $c_{i}$. Moreover, his uplink transmission $P_{i}$ is a parameter that is implicitly determined given the optimal data rate $r_{i}^{*}$ and price $c_{i}^{*}$, while considering a target SINR value $\gamma_{i}^{\text {target }}$, as well as the physical constraints, i.e., $P_{i}^{M i n} \leq$ $P_{i} \leq P_{i}^{\text {Max }}$.

Cornerstone towards solving the above problem under a user-centric approach has been the adoption of pure utility functions which represent in a holistic manner user's degree of satisfaction upon receiving his requested service, while also taking into account user's QoS prerequisites (e.g., target rate) and the respective energy consumption (e.g., transmission power). Additionally, a pricing function with respect to both pricing factor $c_{i}$ and SINR $\gamma_{i}$ matches the level of the price imposed by the WISP and the user's individual willingness to pay for the desired rate based on the relevant service type. It should be noted that we strategically have chosen to express pricing function with respect to SINR, since it better captures and represents user's behavior with respect to the allocation of the system's resources, due to the fact that SINR contains both the physical resources of the uplink transmission power and rate. Therefore, if the user wants to achieve high SINR which is translated into more reliable communication, the price increases representing the fee he should pay in order to enjoy this benefit.

\subsection{Utility function}

In the following, we assume that at every timeslot each user utilizes one type of service, either elastic or inelastic. Towards treating a multi-service wireless networking environment under a unified and common optimization framework, we assume that a net utility function is coupled with each user to reflect his behavior and satisfaction in terms of QoS. To achieve this objective, the introduced function maps the obtained degree of satisfaction to the expected trade-off among the parameters that depict his QoS-related performance, i.e., uplink transmission rate and eagerness to pay the price of resource usage. Hence, each user's net utility function is classified according to the type of service as follows:

$$
U_{i}^{N E T}\left(r_{i}, \mathrm{c}_{i}\right)=\left\{\begin{array}{c}
\frac{r_{i} \cdot f_{i}\left(\gamma_{i}\right)}{P_{i}}-\mathrm{c}_{i}\left(e^{\gamma_{i}}-1\right),(\text { inelastic }) \\
\frac{r_{i} \cdot \log \left(1+f_{i}\left(\gamma_{i}\right)\right)}{P_{i}}-\mathrm{c}_{i}\left(e^{\gamma_{i}}-1\right),(\text { elastic })
\end{array}\right.
$$

With regards to the above relation (2), the efficiency function $f_{i}\left(\gamma_{i}\right)$ represents the successful packet transmission at fixed data rates according to the adopted modulation and coding schemes. The main properties of the efficiency function are: 
1. $f_{i}$ is an increasing function of $\gamma_{i}$.

2. $f_{i}$ is a continuous, twice differentiable sigmoidal function with respect to $\gamma_{i}$.

3. $f_{i}(0)=0$.

4. $f_{i}(\infty)=1$.

In the remaining of the paper for demonstration reasons mainly and without loss of generality, we assume that the efficiency function is of the following form (e.g., $[10,11,25])$.

$$
f\left(\gamma_{i}\right)=\left(1-e^{-A \gamma_{i}}\right)^{M}
$$

where parameter $M$ indicates the number of bits of transmitted packets and $A$ is a parameter influencing the slope of the corresponding curve. For presentation simplicity, in the following, the same modulation scheme is adopted by all users in the cell, which essentially corresponds to the use of a common form of efficiency function by all users.

With reference to the pure utility of users with inelastic services, the numerator $r_{i} \cdot f_{i}\left(\gamma_{i}\right)$ is a sigmoidal function of SINR $\gamma_{i}$, with its inflection point being mapped at the target SINR, $\gamma_{i}^{\text {target }}$ at which his QoS prerequisites are fulfilled. Therefore, if that user's achievable data rate is below inflection point, then a rapid reduction in his utility occurs, a fact that indicates his desire in obtaining and consuming additional system's resources. It should be highlighted that both for the elastic and inelastic services, the numerator of the pure utility function is expressed as a function of the actual transmission rate $r_{i}$ and the efficiency function $f\left(\gamma_{i}\right)$, instead of only the actual transmission data rate that has been adopted so far in the literature. This formulation is proposed in order to better capture users' services' QoS prerequisites, as explained above. The aforementioned formulation of the utility function enables a user to properly regulate his uplink transmission rate and inclination to pay, so as to obtain an SINR level that satisfies his QoS prerequisites.

Now, with reference to the users requesting elastic services, the main characteristic of the latter is their delay-tolerant and high-throughput nature. Hence, the users' goal that request elastic (i.e., non-real time) services is to achieve high throughput, thus increasing their perceived satisfaction, i.e., utility. Based on this observation, users' pure utility function is strictly increasing with respect to the achievable data rate. Specifically, the respective numerator in their pure utility function represents the achievable data rate and is reflected via a log-based strictly increasing function of the user's efficiency function $f_{i}\left(\gamma_{i}\right)$. This formulation is well aligned with the above mentioned high throughput expectations of users requesting elastic services, due to their greedy behavior, essentially aiming at exploiting the network's available spectrum to its full extent, in line however with the physical limitations of the system. In principle, the selected utility function for elastic services has been designed following the Shannon like log-based function paradigm, indicating the maximum data rate under which a transmission can successfully take place for a network's spectral capacity under interference conditions. Consequently, an increase in their obtained data rate results in higher SINR values as a consequence of the nature of function $r_{i} \cdot \log \left(1+f_{i}\left(\gamma_{i}\right)\right)$ which is strictly increasing with respect to $\gamma_{i}$, and therefore, the overall resulting satisfaction as perceived by the users, i.e., $U_{i}^{N E T}$, increases as well.

\section{S-modular games}

Aiming at providing a generic and stable solution for rate allocation alongside price customization, the theory of multi-variable S-modular games emerges as a concrete tool towards showing the trade-off between the involved parameters. Topkis [28] and Vives [26] have introduced the S-modular games, which include the supermodular and submodular game subclasses. The two types of games have exactly opposite characteristics and players' behavior. Regarding supermodular games, an increase in one's player's action for given strategies of the rest of the players reinforces the desire of all the players to increase their actions due to the strategic complementarity. On the opposite side, the strategic substitutes characterize the submodular games, i.e., an increase in one's player's action incentivizes the rest of the players to decrease their actions $[29,30]$. Multi-variable supermodular games as studied in [26] reflect the interrelation between the actions of one user with the rest of the players with whom he competes for the network's scarce resources. Supermodular games have been applied in multiple research works in the literature, which mainly study the resource allocation problem in wireless networks under the assumption that there is only one type of resources to be allocated among the users (especially user's transmission power), thus constituting a single-variable optimization problem. In this paper, we adopt multi-variable S-modular theory in order to solve a two-variable (i.e., rate and price) optimization problem, which asks for different treatment compared to the single variable one and the validity of additional conditions should be examined. S-Modular games guarantee the existence of at least one Nash Equilibrium within multi-space strategy sets $S_{i}=A \times B$, thus surpassing the notions of convexity and differentiability. Furthermore, each player initially adopts his lowest or largest strategy and converges monotonically to an equilibrium, which depends on the initial state. Additionally, if the supermodular game has a unique Nash equilibrium, then it will converge to it, via dominance solvable 
and/or learning rules. In such games, the actions of one player (i.e., higher data rate claim) lead to similar actions from the other users, due to their monotonically increasing best responses $(B R)$ on this player's strategy. The above framework corresponds to the problem of our interest, since a user paying higher prices in order to increase his data rate is followed by a similar trend from the rest of the users, who in order to withstand bandwidth competition will also accept to be charged at higher prices so as to maintain their achieved data rates. Subsequently, we discuss the fundamental properties of a supermodular game, which stands for a two-variable (i.e., $\vec{a}, \vec{b}$ ) optimization problem.

Definition 1 Given a game $G=[N,\{A, B\},\{U(\vec{a}, \vec{b})\}]$, a smooth function $U: A \times B \rightarrow \mathbb{R}$ is supermodular in $\left(a_{i}, b_{i}\right)$ if and only if, [26]:

$$
\frac{\partial^{2} U(\vec{a}, \vec{b})}{\partial a_{i} \partial b_{i}} \geq 0, \forall i
$$

Definition 2 A smooth function $U: A \times B \rightarrow \mathbb{R}$ (i.e., a function that has derivatives of all orders everywhere in its space $A \times B)$ has non-decreasing differences in $\left(a_{i}, b_{i}\right)$, $\left(\overrightarrow{a_{-i}}, \overrightarrow{\mathrm{b}_{-i}}\right)$, if $[26]$ :

$$
\frac{\partial^{2} U(\vec{a}, \vec{b})}{\partial a_{i} \partial a_{j}} \geq 0, \forall j \neq i
$$

given that:

$$
\frac{\partial^{2} U(\vec{a}, \vec{b})}{\partial a_{i} \partial b_{j}}=0, \forall j \neq i
$$

where $\overrightarrow{a_{-i}}$ and $\overrightarrow{b_{-i}}$ are the strategies of all players except for $i$ in the domains $A$ and $B$, respectively.

Theorem 1 A game $G$ is supermodular if the following conditions are satisfied:

i. $\quad i \in N, S_{i}=A_{i} \times B_{i}$ is a compact subset of the Euclidean space

ii. $U$ is supermodular in $\left(a_{i}, b_{i}\right)$,

iii. $U$ has non-decreasing differences in $\left(a_{i}, b_{i}\right)$,

If the game is supermodular, then it has at least one Nash equilibrium.

The proof of this theorem can be found in $[26,31,32]$.

\section{Problem formulation and solution}

\subsection{Rate and price control game}

Game theory has been promoted as a natural and powerful choice when dealing with users that exhibit selfish and greedy behavior. When operating in a competitive environment, the wireless network users act as players that contend against each other and select a strategy space of data rate and willingness to pay, i.e., price, and as a result of their choices achieve a payoff expressed by their utility. In this section, we model our problem as a two-variable (rate $\left(r_{i}\right)$ and price $\left(c_{i}\right)$ )-RP game, assuming a wireless network consisting of $|N|$ users.

Given that the objective of each user is to maximize his utility, the RP game can be formulated as a distributed utility maximization problem. We denote the RP game $G_{R P}=\left[N,\left\{R_{i}, C_{i}\right\},\left\{U_{i}^{N e t}(\vec{r}, \vec{c})\right\}\right]$ as:

$$
\begin{aligned}
& \max _{r_{i} \in R_{i}, c_{i} \in C_{i}} U_{i}^{\text {Net }}(\vec{r}, \vec{c}), \forall i \in N \\
& \text { s.t. } r_{i}^{M i n} \leq r_{i} \leq r_{i}^{M a x} \text { and } c_{i}^{M i n} \leq c_{i} \leq c_{i}^{\text {Max }}
\end{aligned}
$$

where $\vec{r}=\left(r_{1}, \ldots, r_{i}, \ldots, r_{|N|}\right)$ and $\vec{c}=\left(c_{1}, \ldots, \mathrm{c}_{i}, \ldots, \mathrm{c}_{|N|}\right)$ are the vectors of users' uplink transmission rate and prices, respectively, and $R_{i}, C_{i}$ denote the $i_{t h}$ user's strategy spaces in uplink transmission rate and price, respectively. The power consumption of the users considering their optimal uplink transmission data rate $\overrightarrow{r^{*}}$ and customized price $\overrightarrow{c^{*}}$ will be discussed in detail in Section 4.2. The RP game should conclude to a solution that determines the optimal equilibrium (Nash equilibrium) for the system, based on the individual decisions of each user, given the decisions made by the rest of the users. At the Nash equilibrium, no user has the motivation to alter his choice, for the reason that he cannot unilaterally enhance his own utility by applying changes to his personal strategy, given the selections already made by the rest of the users.

Definition 3 The strategy vector $\left(\overrightarrow{r^{*}}, \overrightarrow{c^{*}}\right)=\left(r_{1}^{*}, \ldots, r_{i}^{*}, \ldots\right.$, $\left.r_{|N|}^{*}, c_{1}^{*}, \ldots, c_{i}^{*}, \ldots, \mathrm{c}_{|N|}^{*}\right)$ in the strategy sets $r_{i}^{*} \in R_{i}$ and $c_{i}^{*} \in C_{i}$ is a Nash equilibrium of the RP game if for every user $\forall i$, $i \in N$, the following condition is satisfied:

$$
U_{i}^{N e t}\left(r_{i}^{*}, c_{i}^{*}, \overrightarrow{r_{-i}}, \overrightarrow{c_{-i}}\right) \geq U_{i}^{N e t}\left(r_{i}, c_{i}, \overrightarrow{r_{-i}}, \overrightarrow{c_{-i}}\right)
$$

for all $r_{i} \in R_{i}$ and $c_{i} \in C_{i}$.

Moreover, it should be noted that the existence of Nash equilibrium point guarantees a stable outcome of the RP game while, on the other hand, the nonexistence of such an equilibrium is interpreted as an unstable state of the system. The proof of the existence of at least one Nash equilibrium of RP game is mainly based on the use of supermodular games properties.

Theorem 2: The RP game $G_{R P}$ is supermodular in $\left(r_{i}\right.$, $\left.c_{i}\right)$, if $\forall i, i \in N$ :

$$
\gamma_{i} \geq 0
$$

Proof: The RP game $G_{R P}=\left[N,\left\{R_{i}, C_{i}\right\},\left\{U_{i}^{\text {Net }}(\vec{r}, \vec{c})\right\}\right]$ is supermodular $\forall i \in N$ if the following apply: 
i. $S_{i}=R_{i} \times C_{i}$ is a compact subset of the Euclidean space

ii. $U_{i}^{\text {Net }}$ is smooth and supermodular in $\left(r_{i}, c_{i}\right)$, i.e.,

$$
\frac{\partial^{2} U_{i}^{N e t}}{\partial r_{i} \partial c_{i}} \geq 0, \forall i \in N
$$

iii. $U_{i}^{\text {Net }}$ has non-decreasing differences in $\left(r_{i}, c_{i}\right)$, $\left(\vec{r}_{-i}, \overrightarrow{\mathrm{c}}_{-i}\right)$, if:

$$
\frac{\partial^{2} U_{i}^{N e t}}{\partial r_{i} \partial r_{j}} \geq 0, \forall j \neq i
$$

given that:

$$
\frac{\partial^{2} U_{i}^{N e t}}{\partial r_{i} \partial c_{j}}=0, \forall j \neq i
$$

In a supermodular game, there always exists external equilibria. A largest element $\left(\overline{r_{i}}, \overline{c_{i}}\right)=\sup \left\{\left(r_{i}, c_{i}\right) \in S_{i}\right.$ $\left.: B R\left(r_{i}, c_{i}\right) \geq\left(r_{i}, c_{i}\right)\right\}$ and a smallest element $\left(\underline{r_{i}}, \underline{c_{i}}\right)=\inf$ $\left\{\left(r_{i}, c_{i}\right) \in S_{i}: B R\left(r_{i}, c_{i}\right) \leq\left(r_{i}, c_{i}\right)\right\}$, with $B R\left(r_{i}, c_{i}\right)$ denoting the best response strategy of a player given the strategies of the rest of the involved players. According to [27], $B R$ is standard, thus guaranteeing the convergence of the RP game to its Nash Equilibrium, assuming any feasible initial solution. Combining relations (9)-(11) and requesting to hold true together, we derive the set of values of SINR, under which RP game is supermodular.

Considering the users requesting elastic services and examining the aforementioned two conditions, we have

$$
\begin{gathered}
\frac{\partial^{2} U_{i}^{\text {Net }}}{\partial r_{i} \partial c_{i}}=\frac{e^{\gamma_{i}} \gamma_{i}}{r_{i}} \\
>0, \forall i \in N, r_{i} \in\left[r_{i}^{\text {Min }}, r_{i}^{\text {Max }}\right], P_{i} \in\left[P_{i}^{M i n}, P_{i}^{M a x}\right], \frac{\partial^{2} U_{i}^{\text {Net }}}{\partial r_{i} \partial r_{j}}=0, \text { and } \frac{\partial^{2} U_{i}^{\text {Net }}}{\partial r_{i} \partial c_{j}}=0
\end{gathered}
$$

where $\frac{\partial \gamma_{i}}{\partial r_{i}}=-\frac{\gamma_{i}}{r_{i}}$. Also, considering the users requesting inelastic services, based on Eq. (2) and regarding the two conditions mentioned above, we have

$$
\begin{gathered}
\frac{\partial^{2} U_{i}^{\text {Net }}}{\partial r_{i} \partial c_{i}}=\frac{e^{\gamma_{i}} \gamma_{i}}{r_{i}} \\
>0, \forall i \in N, r_{i} \in\left[r_{i}^{M i n}, r_{i}^{M a x}\right], P_{i} \in\left[P_{i}^{M i n}, P_{i}^{M a x}\right], \frac{\partial^{2} U_{i}^{N e t}}{\partial r_{i} \partial r_{j}}=0, \text { and } \frac{\partial^{2} U_{i}^{N e t}}{\partial r_{i} \partial c_{j}}=0
\end{gathered}
$$

Thus, we conclude that the RP game is supermodular in $\left(r_{i}, c_{i}\right)$ if $\forall i \in N$, the following condition holds true:

$$
\gamma_{i} \geq 0
$$

Based on condition (8), we practically conclude that the RP game is supermodular in the whole strategy space, i.e., $R_{i} \times C_{i}=\left[r_{i}^{M i n}, r_{i}^{M a x}\right] \times\left[c_{i}^{\text {Min }}, c_{i}^{\text {Max }}\right]$. Since the game is confirmed to be supermodular, we conclude that a non-empty set of Nash Equilibria exists as follows:

Theorem 3 There exists a strategy vector $\left(\overrightarrow{r^{*}}, \overrightarrow{c^{*}}\right)$ $=\left(r_{1}^{*}, \ldots, r_{i}^{*}, \ldots, r_{|N|}^{*}, c_{1}^{*}, \ldots, c_{i}^{*}, \ldots, \mathrm{C}_{|N|}^{*}\right)$ within the strategy sets $r_{i}^{*} \in R_{i}$ and $c_{i}^{*} \in C_{i}$ which is a Nash equilibrium for the RP game $\forall i \in N$. Hence, no user can further increase his utility taking as granted the choices of the other users, i.e.,

$$
\left(r_{i}^{*}, c_{i}^{*}\right)=\arg \max U_{i}^{N e t}(\vec{r}, \vec{c}), \forall r_{i} \in R_{i} \text { and } c_{i} \in C_{i}
$$

The proof of Theorem 3 follows the same basic steps as in Theorem 1, [26, 31, 32].

\subsection{Power consumption}

Based on Theorem 3, the users determine their optimal data rates $\overrightarrow{r^{*}}$ and their optimal customized prices $\overrightarrow{c^{*}}$ in a distributed manner, while considering the existing physical and external constraints, i.e., $r_{i}^{\text {Min }} \leq r_{i} \leq r_{i}^{M a x}$ and $c_{i}^{\text {Min }}$ $\leq c_{i} \leq c_{i}^{\text {Max }}$, respectively. In our proposed approach, each user has control over the variables of the data rate $r_{i}$ and the customized price $c_{i}$, which determine his corresponding perceived satisfaction that is appropriately expressed via the adopted net utility function. However, each user has an extra constraint considering his energy-availability, which is appropriately presented via the feasible transmission power interval, i.e., $P_{i}^{\mathrm{Min}} \leq P_{i} \leq$ $P_{i}^{M a x}$. The lower bound $P_{i}^{M i n}$ expresses the minimum necessary transmission power in order user's signal to be demodulated by the receiver. The upper bound $P_{i}^{\text {Max }}$ stems from mobile user's available energy.

In our proposed framework, the power consumption $P_{i}^{*}$ of each user results from his optimal uplink transmission data rate $r_{i}^{*}$ and his target SINR value $\gamma_{i}^{\text {target }}$ based on Eq. (1). It is noted that the power consumption is not constant, but dynamically determined via the control parameters of the system, i.e., optimal data rate $r_{i}^{*}$ and customized price $c_{i}^{*}$. Thus, the power consumption for each user is determined as follows:

$$
P_{i}^{*}=\min \left(\max \left(\frac{\gamma_{i}^{t} \text { arget }}{W G_{i}} r_{i}^{*}\left(\sum_{j=i+1}^{|N|} G_{j} P_{j}^{*}+I_{0}\right), P_{i}^{M i n}\right), P_{i}^{M a x}\right)
$$

It should be clarified that the RP game may have multiple NEs. However, each mobile user selects the NE point (12) which concludes to his minimum 
corresponding power consumption, as determined in (13), in order to improve his achieved energy efficiency.

\section{Convergence and RP algorithm}

\subsection{Convergence}

In this section, we study and prove the convergence of the RP game to a Nash equilibrium point, which constitutes a stable solution. The proof is based on the use and properties of best response dynamics, by concluding that users' net utility function is a standard one. User's best response strategy in the Euclidean space $R_{i} \times C_{i}=$ [ $\left.r_{i}^{M i n}, r_{i}^{M a x}\right] \times\left[c_{i}^{M i n}, c_{i}^{M a x}\right]$ is expressed as follows:

$$
\begin{aligned}
B R_{i}\left(r_{i}, \mathrm{c}_{i}\right) & =\left(r_{i}{ }^{*}, \mathrm{c}_{i}{ }^{*}\right) \\
& =\underset{\substack{r_{i} \in R_{i} \\
c_{i} \in C_{i}}}{\arg \max } U_{i}^{N E T}(\vec{r}, \vec{c})
\end{aligned}
$$

Theorem 4 The RP game $G_{R P}$ converges to its Nash equilibrium point starting from any initial feasible solution, (i.e., $\left.\left(r_{i}^{(0)}, \mathrm{c}_{i}^{(0)}\right)\right) ; r_{i}^{\text {Min }} \leq r_{i} \leq r_{i}^{\text {Max }}$ and $c_{i}^{\text {Min }} \leq c_{i}^{(0)} \leq c_{i}^{\text {Max }}$, provided that user's best response strategy is a standard function.

Proof: The basic step to prove that RP game converges to its Nash equilibrium $\left(\overrightarrow{r^{*}}, \overrightarrow{c^{*}}\right)=\overrightarrow{B R}(\vec{r}, \vec{c})$ is to show that user's best response strategy belongs to the family of standard functions [27]. A function $f$ is indicated as standard, if the following properties hold:

i. Positivity: $f(x)>0$;

ii. Monotonicity: if $\mathrm{x} \geq \mathrm{x}^{\prime}$, then $f(\mathrm{x}) \geq f\left(\mathrm{x}^{\prime}\right)$ and

iii. Scalability: for all $a>1, a f(x) \geq f(a x)$,

for all $\mathrm{x} \geq 0$, where $\mathrm{x}=\left(x_{1}, x_{2}, \ldots, x_{N}\right)$ is a Nash equilibrium point. With reference to our RP game, we readily observe that all the above properties hold true, as shown below:

i. $(\vec{r}, \vec{c})>0$, thus $\overrightarrow{B R}(\vec{r}, \vec{c})>0$, via Eq. (14),

ii. if $(\vec{r}, \vec{c})>\left(\vec{r}^{\prime}, \vec{c}^{\prime}\right)$, then via (14), we conclude that $\overrightarrow{B R}(\vec{r}, \vec{c})>\overrightarrow{B R}\left(\vec{r}^{\prime}, \vec{c}^{\prime}\right)$,

iii. for all $a>1$, since $\overrightarrow{B R}(\vec{r}, \vec{c})$ is a strictly increasing function with respect to $(\vec{r}, \vec{c})$, then it follows that $a \overrightarrow{B R}(\vec{r}, \vec{c})>\overrightarrow{B R}(a \vec{r}, a \vec{c})$.

\subsection{RP algorithm}

In the following, we present a low complexity algorithm associated with the convergence to the Nash equilibrium of the RP game, which operates in an iterative and distributed manner. Besides providing a stable and universally applicable solution, the RP algorithm integrates within the same scheme both the rate allocation as well as the price setting for each user at the moment of receiving the required service. The algorithm is referred to as RP algorithm. The descriptive/pseudo-code steps of the RP algorithm are defined as follows.

\subsubsection{RP algorithm}

Step 1The feasible pricing factor sets $\left[c_{i}^{M i n}, c_{i}^{\text {Max }}\right]$ are disclosed by the WISP to the users. Each user selects a random initial feasible uplink transmission power $P_{i}^{(i t e=0)} \in\left[P_{i}^{M i n}, P_{i}^{M a x}\right]$ for the initialization of the transmission. Set ite $=0$, where ite represents the number of iterations.

Step 2The users, given the network's physical constraints, define their optimal data rate $r_{i}^{*} \in[$ $\left.r_{i}^{M i n}, r_{i}^{M a x}\right]$ and their willingness to pay, i.e., price $c_{i}^{*} \in\left[c_{i}^{\text {Min }}, \mathrm{c}_{i}^{\text {Max }}\right]$, as in Eq. (14). Set ite ite +1 .

Step 3The WISP broadcasts the imposed interference to all users $\sum_{j=1}^{N} G_{j} P_{j}$ and each user $i$ defines his sensed interference, as described in the NOMA technique.

Step 4The users compute their respective transmission power $P_{i}^{i t e+1} \in\left[P_{i}^{M i n}, P_{i}^{M a x}\right]$ given their predetermined/target SINR values and the optimal data rate $r_{i}^{*}$ based on Eq. (13).

Step 5tf the pure utilities of each one of the users do not improve $U_{i}\left(r_{i}^{i t e}, c_{i}^{i t e}\right)>U_{i}\left(r_{i}^{i t e+1}, c_{i}^{i t e+1}\right)$, terminate; else go to Step 2.

A key property of the RP algorithm is its distributed operation, owing to the fact that the final decisions are concluded by each mobile user individually. Each mobile user takes the decision about his best response strategy based on his own available personal information, i.e., channel gain $G_{i}$, and a global information broadcasted by the base station, i.e., overall interference $\sum_{j=1}^{N} G_{j} P_{j}$. Therefore, in the RP algorithm, the message exchange overhead is rather limited. It should be clarified that, at Step 3, the base station simply announces the total interference to the users. The task of deciding the uplink transmission data rate and price is performed by the users themselves. Thus, the only message exchange overhead from the base station to the mobile users is the broadcasting of the values $c_{i}^{\mathrm{Min}}$, $\mathrm{c}_{i}^{\mathrm{Max}}$ at the beginning of the RP algorithm's execution and the total interference at each iteration of the algorithm. Also, it is highlighted that the base station broadcasts the aforementioned information, thus the users are not burdened with additional signaling exchange. Therefore, the complexity of the algorithm is not impacted by an increase on the number of the users served by the cell. Additional results regarding the computational time and complexity of various implementations of the proposed $\mathrm{RP}$ framework are presented below in Section 6. 


\section{Performance evaluation}

In this section, the performance evaluation of the proposed framework is conducted through modeling and simulation. Indicative numerical results are presented to gain some insight about and demonstrate the key properties and advantages of the introduced framework and the RP algorithm. In order to better appreciate and comprehend the benefits and overall operational efficiency of the proposed approach, its performance achievements and improvements are demonstrated via a comparative evaluation study against relevant state of the art research works existing in the literature.

Initially, in order to adapt the RP framework into different resource allocation scenarios and obtain insightful, comparative results and show how the adopted pricing scheme may influence the equilibrium point, four different pricing policies have been considered:

(a) Customized pricing, where each user separately declares the price he would accept in order to achieve the desired QoS upon receiving the required service,

(b) Central pricing, where the WISP charges all users with a common price for their transmission,

(c) Service pricing, where the users are priced according to their requested service type, and lastly,

(d) Zone pricing, where several non-overlapping rings (zones) are created within each cell, while within each zone homogeneous pricing, is applied to all users belonging to that zone.

One of the advantages of introducing price customization is the ability to segment the users into groups according to specific criteria (e.g., service, location). Hence, the selected pricing mechanisms serve different purposes depending on the priorities of the users and/or the WISP regarding complexity and computational resource consumption reduction, interference mitigation, or the utilization of network's capacity.

Furthermore, the aforementioned different versions of the RP algorithm are compared against [10], where data rates are jointly allocated among the users alongside transmission powers, and [15], where the system is designed towards maximizing its uplink sum rate. For fairness in the comparison, the approaches proposed in $[10,15]$ have properly been adopted to the NOMA access scheme.

\subsection{Model and assumptions}

Throughout our evaluation analysis, the uplink of a single cell system is considered where users place requests for elastic and inelastic services. To study the behavior of the proposed RP framework under different network sizes, we varied the number of users $|N(t)|$ from 10 to 40 , while they were assumed to be randomly placed within the cell of radius $R=1000 m$ for each simulation scenario. For any considered network topology, the order of requested services was alternating among the users, in the sense that the closest to the base station user requests inelastic service, the second closest requests elastic service, and so on. Such a setting allows for a balanced distribution, both physical (location) and logical (type of service), of the services and users in the system. Users requesting inelastic services are assumed to have service rate target of $96 \mathrm{Kbps}$ (e.g., simple video upload). For statistical purposes, a Monte Carlo simulation was adopted, where for all the presented results we studied 10,000 random topologies considering randomly generated users' positions. Users' path gains are modeled through the simplified path loss model $G_{i}=K_{i} / d_{i}^{a}$, where $d_{i}$ stands for the distance between user $i$ and the serving base station, $a$ expresses the distance loss exponent (e.g., $a=4$ ), and $K_{i}$ is a log-normal distributed random variable with mean 0 and variance $\sigma^{2}=8 \mathrm{db}$, representing shadow effect. For simplicity in the presentation and without loss of generality, all users are assumed to adopt the same efficiency function $f\left(\gamma_{i}\right)$ $=\left(1-e^{-1.15 \cdot \gamma_{i}}\right)^{M}, M=80$, simply reflecting the use of the same modulation and coding scheme by all users.

For practical purposes, we assume that every user imposes a strict constraint on his maximum uplink transmission power, i.e., $P_{i}^{M a x}=0.1 \mathrm{Watt}$, and the WISP has specific pricing boundaries that announces ranging between a maximum and minimum price, i.e., $c_{i}^{\operatorname{Max}}=2 \cdot 10^{6}, c_{i}^{M i n}$ $=0$. Throughout our numerical evaluation all the relevant parameters, including $A, M$ parameters of $f\left(\gamma_{i}\right)$ and the bounds $c_{i}^{M a x}, c_{i}^{M i n}$ have been appropriately chosen and configured, so as to be realistic on one hand, while on the other hand to ensure almost the same order of magnitude for both terms of user's net utility function $U_{i}^{N E T}$.

\subsection{Numerical results and discussions}

Figures 1 and 2 present the performance of the considered six approaches (i.e., the four alternatives of the RP framework, and the approaches in $[10,15])$, in terms of users' average uplink transmission rate achieved and average energy efficiency, respectively, versus the network size. It is noted that the energy efficiency refers to the number of efficiently transmitted bits per Joule of energy consumed and is measured as the ratio of user's achievable transmission rate over the used transmission power. The results clearly reveal the superiority of all four alternatives of the proposed RP framework in terms of both high achievable data rate and energy efficiency. This enhanced performance stems from the fact that under RP approach the user has more degrees of freedom, i.e., data rate and price, as well as the intelligence of determining them dynamically and in an optimal way, as the system evolves. Furthermore, the achieved high 


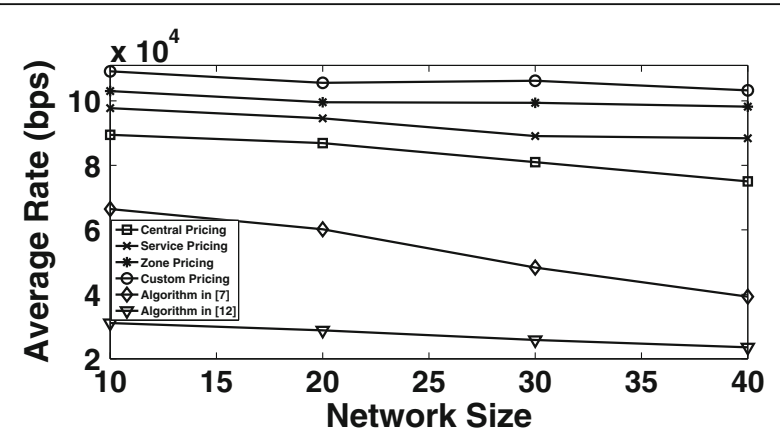

Fig. 1 Users' average uplink transmission data rate versus the number of users considering RP framework and comparative scenarios

energy efficiency represents in a combined metric the objective of simultaneously achieving lower transmission power and higher data rate. Moreover, based on the presented numerical results, it becomes evident that the RP framework performs well as the network grows in size, while the rest of the considered frameworks, e.g., [10, 15], present significantly lower data rate and energy efficiency, and in some cases even collapse due to the absence of control over the corresponding price that the end user is penalized by the WISP. The customized pricing policy proposed in our framework outperforms the rest of the implementation alternatives, i.e., zone, service, and central pricing, in terms of increased data rate and energy efficiency awards, due to its attribute to adapt well to each user's QoS prerequisites and willingness to pay in order to fulfill them. The results with reference to the rest of the pricing alternatives of our framework present a trade-off among the achievable data rate, energy efficiency, and computational complexity when compared to the customized pricing policy. Specifically, zone-based pricing policy presents a well-balanced alternative implementation both in terms of efficiency and effectiveness, as it achieves an improvement in the computational time efficiency of approximately 28\% when compared to the custom pricing implementation

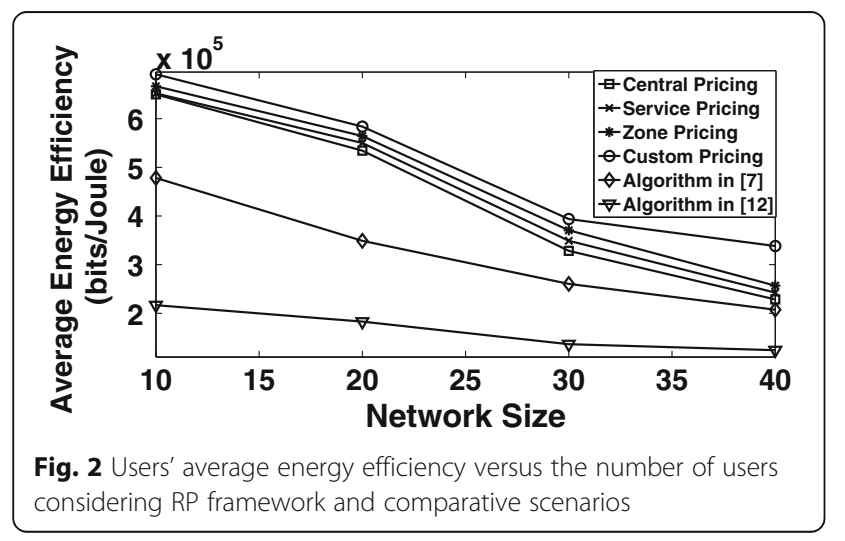

without paying any significant cost as far as the achieved data rate and energy efficiency is concerned. On the other hand, the rest of the implementation alternatives, i.e., central and service pricing, achieve to further improve the computational time efficiency by 98 and $90 \%$ compared to the custom pricing implementation, paying the penalty however of increased losses in the obtained data rate (i.e., 28.08 and $14.85 \%$, respectively) and energy efficiency (i.e., 20.91 and $16.14 \%$, respectively). Comprehensive comparative results with respect to the metric of computation time efficiency of all the considered approaches, as compared to the baseline scenario of custom pricing implementation, are presented in Table 1. Based on these results, it is highlighted that a practical potential limitation of the custom pricing policy is its increased computational time compared to the alternative heuristic approaches (i.e., central, service and zone pricing), as well as compared to [10, 15]. Additionally, we have extended our study to the computational complexity per iteration of each of the comparative approaches, which is quantified via the average computational time per iteration per user, given the distributed nature of the algorithms. The results are presented in the last column of Table 1 as well. We observe that the average computational time per iteration per user for the custom pricing implementation is quite low; however, given the two-variables optimization problem that is solved, we need more iterations to converge to the optimal solution. This is the reason that the overall computational time of the custom pricing framework is increased compared to the other approaches, as discussed above.

Figures $3 \mathrm{a}, \mathrm{b}$ and $4 \mathrm{a}, \mathrm{b}$ present similar results as in Figs. 1 and 2-reflecting average uplink transmission rate (Fig. 3a, b) and energy efficiency (Fig. 4a, b) -separately for the users that request inelastic and elastic services, respectively, in order to gain insightful information about the influence of the type of requested service on the equilibrium values of data rate and pricing. It is noted that for each simulated scenario considering increasing number of users from 10 to 40 , half of them

Table 1 Comparative results for computation time efficiency and average time per iteration per user

\begin{tabular}{lll}
\hline Scenario & $\begin{array}{l}\text { Increase in average } \\
\text { computation } \\
\text { time efficiency } \\
\text { (against custom price) }\end{array}$ & $\begin{array}{l}\text { Average time per } \\
\text { iteration per user } \\
\text { (seconds) }\end{array}$ \\
\hline$[15]$ & $97.67 \%$ & 0.005200 \\
{$[10]$} & $75.97 \%$ & 0.031000 \\
RP: central price & $97.56 \%$ & 0.001800 \\
RP: service price & $90.81 \%$ & 0.000792 \\
RP: zone price & $21.81 \%$ & 0.000699 \\
RP: custom price & (Baseline scenario) & 0.000693 \\
\hline
\end{tabular}




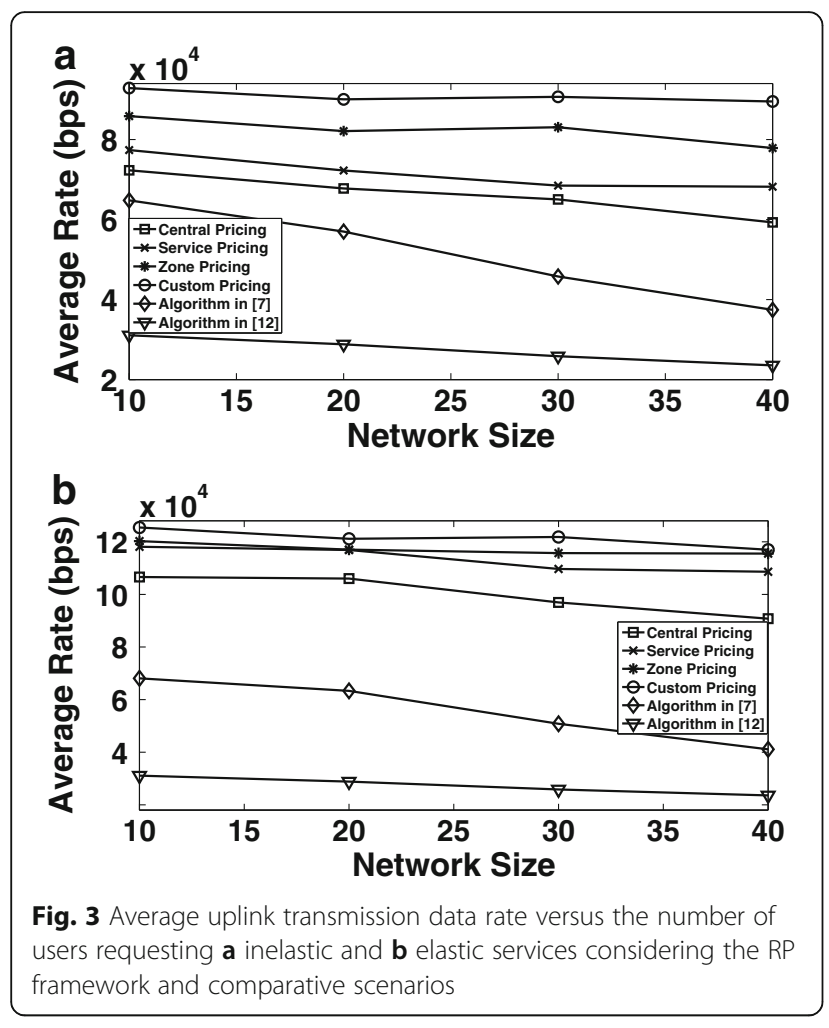

request inelastic services and the rest of them elastic services. Observing the results of Figs. $3 a, b$ and $4 a, b$, we conclude that the custom pricing implementation of RP algorithm outperforms both the rest of the implementation alternatives of RP algorithm (i.e., zone, central, service pricing), as well as the alternatives in the literature (i.e., $[10,15]$ ), in terms of increased achieved data rate and energy efficiency for any type of user, i.e., irrespective of the requested service type (i.e., inelastic or elastic).

In Table 2, we summarize the average percentage increase in terms of achievable data rate and energy efficiency for each algorithm, considering the six comparative scenarios. Based on these results, we observe that the different implementations of the RP algorithm considerably improve the attainable rates and energy efficiency, achieving approximately up to $292 \%$ increase of data rate and 203\% increase of energy efficiency when compared to [15].

As mentioned before, the performance improvement of the RP implementation with customized pricing is achieved at the cost of increased computational complexity. The convergence time of the RP algorithm can be further improved via adopting several operational considerations and enhancements as discussed below. Initially, in order to derive the presented results, we execute the RP algorithm per timeslot. Towards improving the convergence performance, we can execute the RP algorithm considering as initial value of users'

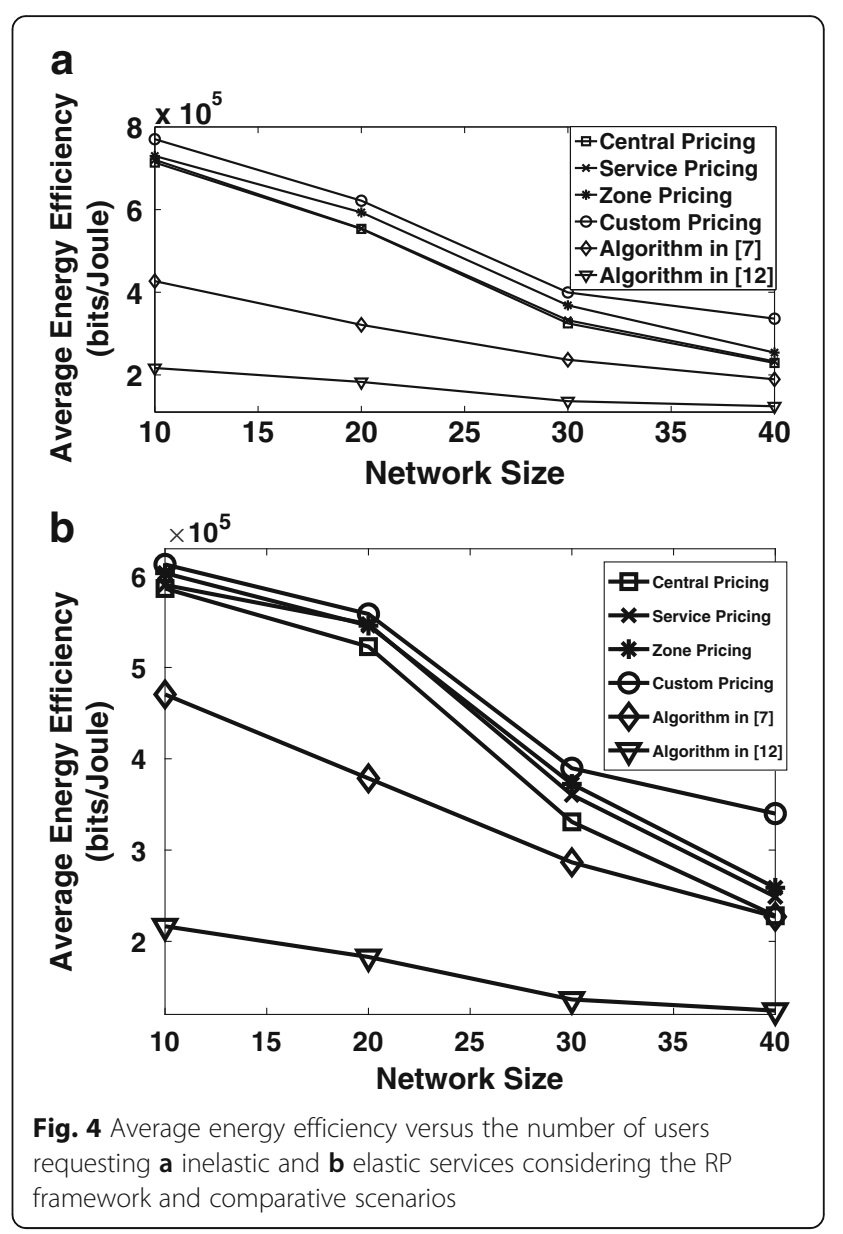

transmission data rate and price the ones that were calculated in the previous timeslot, i.e., "educated" implementation of RP algorithm. Taking into consideration that in reality the examined wireless communication environment is not expected to undergo significant changes within the duration of few timeslots, for all practical purposes, the users' uplink transmission rate and price values as obtained by the RP framework could be applicable to several consecutive timeslots, thus further reducing the corresponding implementation overhead.

Table 2 Comparative results for average sum rate and energy efficiency of various comparative scenarios against [15]

\begin{tabular}{lll}
\hline Scenario & Increase in average sum rate & $\begin{array}{l}\text { Increase in average } \\
\text { energy efficiency }\end{array}$ \\
\hline$[15]$ & - & - \\
{$[10]$} & $94 \%$ & $33.15 \%$ \\
RP: central price & $205.29 \%$ & $163.83 \%$ \\
RP: service price & $240.61 \%$ & $171.76 \%$ \\
RP: zone price & $269.58 \%$ & $180.10 \%$ \\
RP: custom price & $291.71 \%$ & $202.62 \%$ \\
\hline
\end{tabular}




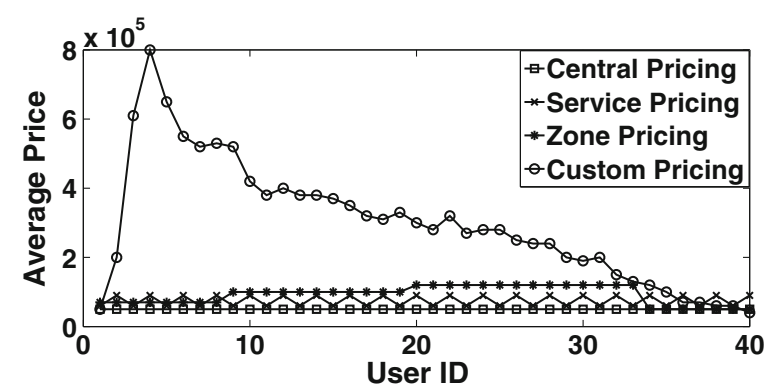

Fig. 5 Average price (dimensionless) versus user's ID considering the alternative implementations of RP framework

Figure 5 represents the actual values of price $c_{i}$ imposed to the users as a function of user's ID considering the four pricing alternatives of RP framework, i.e., (i) custom, (ii) zone, (iii) service, and (iv) central pricing. It should be noted that for demonstration purposes only, the price $c_{i}$ is a dimensionless quantity. However, in a practical implementation of RP framework, the price $c_{i}$ could be mapped to real monetary units. The prices that are presented in Fig. 5 are the Nash equilibrium values of RP algorithm for each user. Considering the service pricing implementation, the results reveal that the users who request elastic service conclude to higher prices due to their need to achieve higher uplink transmission rates. On the other hand, in the zone pricing implementation, it is noted that the less distant users, i.e., first zone, are penalized less due to their good channel gain conditions that conclude to high transmission data rate with lower cost. Additionally, in order to account for the disadvantage of the more distant users due to their deteriorated channel conditions, these users are assigned lower prices as their distance increases. The custom pricing also follows the same concept (i.e., relation of pricing and users' channels' conditions/distance from the base station). Finally, the central pricing implementation alternative of RP algorithm is the less "aggressive" one with respect to the imposed price to the users, due to the fact that it should consider the heterogeneous characteristics (i.e., requested service, channel conditions) of all the users.

Towards examining the behavior of the RP algorithm under the scenario of users' mobility within the cell, we study the impact of user's mobility on his transmission characteristics, i.e., data rate and power, and on his willingness to pay for the requested service, i.e., price. It is highlighted that the duration of a timeslot is $0.5 \mathrm{msec}$ [33]; thus, user's intracell mobility can be considered rather limited per timeslot. As a result, the users can readjust their position coordinates within the cell per timeslot without significantly distorting the resource allocation process. For demonstration purposes, we study the impact of user's mobility to his transmission characteristics for a time-window where the user can move up to $10 \mathrm{~m}$. The results reveal that the change in the rate and price allocation process is minor (approximately $0 \%$, whereas a slight increase in the transmission power is observed (less than $0.1 \%$ ). This implies that the RP algorithm maintains steady outcomes concerning the power and rate allocation, since the users constantly aim to achieve the data rates as close as possible to the feasible upper bounds. Hence, a change in user's location will not affect his data rates requirements, besides a small adjustment to the transmission power levels, so as to claim the same data rate levels. Moreover, the adoption of the NOMA SIC technique maintains the intracell interference to low levels; hence, user's mobility impact within the duration of a timeslot cannot have amplifying impact on the main transmission characteristics of the users. Additionally, by considering stochastic user's mobility, a high degree of offset among new users' positions can take place, with a number of users slightly improving their transmission (e.g., slightly better channel gain conditions) and counterbalance any negative transmission impact from the users who deteriorate their transmission capabilities in the new positions.

\section{Conclusions}

In this article, we tackled the problem of joint rate allocation and customized price setting in NOMA wireless networks supporting users with heterogeneous QoS requirements, following a user-centric paradigm. Through the use of a net utility function with two variables, the satisfaction of each user is highlighted as the trade-off between his desired data rates and the price he is charged in order to receive the respective service. The above optimization problem has been properly formulated as a non-cooperative supermodular game, its solution was obtained, and its convergence to its Nash equilibrium point was shown, while an iterative algorithm is devised to obtain it in a distributed manner.

The performance of this framework was in depth examined through a series of simulation experiments including different pricing policies, whereas the superiority of the proposed approach was clearly demonstrated by comparing it with other research works in the literature. Specifically, it has been shown that the different implementations of the $\mathrm{RP}$ algorithm succeed in providing considerably higher data rates in all different user classes while at the same time maintaining transmission power at low levels. This was achieved by applying customized pricing schemes to each user/user groups, thus precisely extracting consumer surplus regarding the QoS prerequisites. Also, due to the flexible and generic two parameter utility-based design of the described work, the proposed problem solution can be applied in various allocation problems for different resources, network topologies, access technologies, etc. 


\subsection{Method}

This paper studies the combined problem of rate allocation and customized price setting, in NOMA wireless networks supporting users with heterogeneous QoS requirements, following a user-centric paradigm. The performance of the proposed framework was in depth examined through a series of simulation experiments including different pricing policies, whereas the superiority of the proposed approach was clearly demonstrated by comparing it with other research works in the literature. Specifically, it has been shown that the different implementations of the proposed algorithm succeed in providing considerably higher data rates in all different user classes while at the same time maintaining transmission power at low levels. The simulation code was written in Matlab.

\section{Abbreviations}

BR: Best response; BS: Base station; ISPs: Internet service providers; NOMA: Non-orthogonal multiple access; QoS: Quality of service; RP: Rate and price; SIC: Successive interference cancelation; SINR: Signal-to-interferenceplus-noise ratio; WISPs: Wireless Internet service providers

\section{Acknowledgements}

The research of Dr. Eirini Eleni Tsiropoulou was partially supported by the UNM Research Allocation Committee award.

Availability of data and materials

The source code and generated data are the authors' intellectual property.

\section{Authors' contributions}

All authors contributed significantly to the research work presented in this paper. PV had a leading role in the formulation and solution of the considered optimization problem, while performing a detailed evaluation and analysis of the developed algorithms, through conducting an extensive set of simulations. EET mainly contributed to the use of a game theoretic framework towards solving the optimization problem under consideration, while devoting significant efforts to the comparative performance evaluation, and had key leading role in the article editing and presentation. SP introduced the original concept on which our current work was based, orchestrated the overall development of the performance evaluation work, and had the coordination in the writing of the article. All authors read and approved the final manuscript.

\section{Competing interests}

The authors declare that they have no competing interests.

\section{Publisher's Note}

Springer Nature remains neutral with regard to jurisdictional claims in published maps and institutional affiliations.

\section{Author details}

'School of Electrical \& Computer Engineering, National Technical University of Athens, Zografou, 15773 Athens, Greece. ${ }^{2}$ Department of Electrical \& Computer Engineering, University of New Mexico, Albuquerque, NM 87131, USA.

Received: 25 January 2018 Accepted: 15 May 2018

Published online: 23 May 2018

\section{References}

1. M Al-Imari, P Xiao, MA Imran, Receiver and resource allocation optimization for uplink NOMA in $5 \mathrm{G}$ wireless networks. Int. Symp. Wirel. Commun. Sys. 2015, 151-155 (2015)

2. Q Song, L Nuaymi, $X$ Lagrange, Survey of radio resource management issues and proposals for energy-efficient cellular networks that will cover billions of machines. EURASIP J. Wirel. Commun. Netw. 2016, 140 (2016). https://doi.org/10.1186/s13638-016-0636-y

3. J Choi, On the power allocation for a practical multiuser superposition scheme in NOMA systems. IEEE Commun. Lett. 20(3), 438-441 (2016)

4. EE Tsiropoulou, P Vamvakas, S Papavassiliou, Joint customized price and power control for energy-efficient multi-service wireless networks via Smodular theory. IEEE Trans. Green Commun. Netw. 1(1), 17-28 (2017)

5. M Rasti, AR Sharafat, B Seyfe, Pareto-efficient and goal-driven power control in wireless networks: a game-theoretic approach with a novel pricing scheme. IEEE/ACM Trans. Netw. 17(2), 556-569 (2009)

6. MR Musku, AT Chronopoulos, DC Popescu, Joint rate and power control using game theory. IEEE Cons. Commun. Netw. Conf. (2), 1258-1262 (2006)

7. MR Musku, AT Chronopoulos, DC Popescu, Joint rate and power control with pricing. GLOBECOM '05. IEEE Glob. Tel. Conf. (6), 5-3470 (2005)

8. MR Musku, AT Chronopoulos, DC Popescu, A Stefanescu, A game-theoretic approach to joint rate and power control for uplink CDMA communications. IEEE Trans. Commun. 58(3), 923-932 (2010)

9. AT Chronopoulos, MR Musku, S Penmatsa, DC Popescu, Spectrum load balancing for medium access in cognitive radio systems. IEEE Commun. Lett. 12(5), 353-355 (2008)

10. EE Tsiropoulou, P Vamvakas, S Papavassiliou, Joint utility-based uplink power and rate allocation in wireless networks: a non-cooperative game theoretic framework. Elsevier Phys. Commun. J. 9, 299-307 (2012)

11. EE Tsiropoulou, P Vamvakas, S Papavassiliou, Supermodular game-based distributed joint uplink power \& rate allocation in two-tier SC-FDMA femtocell networks. IEEE Trans. Mob. Comp. 16 (9), 2656-2667 (2016). https://doi.org/10.1109/TMC.2016.2622263

12. $Y$ Zhang, HM Wang, $Q$ Yang, $Z$ Ding, Secrecy sum rate maximization in nonorthogonal multiple access. IEEE Commun. Lett. 20(5), 930-933 (2016)

13. Z Na, X Li, X Liu, Z Deng, Subcarrier allocation based simultaneous wireless information and power transfer for multiuser OFDM systems. EURASIP J. Wirel. Commun. Netw. 2017, 148 (2017). https:/doi.org/10.1186/s13638-017-0932-1

14. C Yang, S Jordan, Power and rate allocation for video conferencing in cellular networks. EURASIP J. Wirel. Commun. Netw. 2013, 31 (2013). https://doi.org/10.1186/1687-1499-2013-31

15. S Papavassiliou, C Li, Joint throughput maximization and fair uplink transmission scheduling in CDMA systems. EURASIP J. Wirel. Commun. Netw. 2009 (1), 1-15 (2009)

16. Q Sun, S Han, Z Xu, S Wang, I Chih-Lin, Z Pan, Sum rate optimization for MIMO non-orthogonal multiple access systems. IEEE Wirel. Commun. Netw. Conf. 2015, 747-752 (2015)

17. S Shakkotai, R Srikant, A Ozdaglar, D Acemoglu, The price of simplicity. IEEE J. Sel. Areas Commun. 26 (7), 1269-1276 (2008)

18. CK Chau, Q Wang, DM Chiou, On the viability of Paris metro pricing for communication and service networks. IEEE INFOCOM. 2010, 1-9 (2010)

19. S Ha, S Sen, C Joe-Wong, Y Im, M Chiang, TUBE: time-dependent pricing for mobile data. ACM SIGCOMM. 42 (4), 247-258 (2012)

20. L Zheng, C Joe-Wong, CW Tan, S Ha, M Chiang, Customized data plans for mobile users: feasibility and benefits of data trading. IEEE J. Sel. Areas Commun. 35(4), 949-963 (2017)

21. M Andrews, Understanding the effects of quota trading on mobile usage dynamics. IEEE WiOpt. 2016, 1-8 (2016)

22. X Gong, L Duan, $X$ Chen, J Zhang, When social network effect meets congestion effect in wireless networks: data usage equilibrium and optimal pricing. IEEE J. Sel. Areas Commun. 35(2), 449-462 (2017)

23. NC Luong, P Wang, D Niyato, Y Wen, Z Han, Resource management in cloud networking using economic analysis and pricing models: a survey. IEEE Commun. Surv. Tutorials 19(2), 954-1001 (2017)

24. NC Luong, DT Hoang, P Wang, D Niyato, DI Kim, Z Han, Data collection and wireless communication in internet of things (loT) using economic analysis and pricing models: a survey. IEEE Commun. Surv. Tutorials 18(4), 2546-2590 (2016)

25. L Gao, G losifidis, J Huang, L Tassiulas, Hybrid data pricing for networkassisted user-provided connectivity. IEEE INFOCOM. 2014, 682-690 (2014)

26. X Vives, Complementarities and games: new developments. J. Econ. Lit. XLIII, 437-479 (2005)

27. RD Yates, A framework for uplink power control in cellular radio systems. IEEE J. Sel. Areas Commun. 13, 1341-1347 (1995)

28. DM Topkis, Equilibrium points in nonzero-sum n-person submodular games. SIAM J. Control. Optim. 17(6), 773-787 (1979)

29. D Fudenberg, J Tirole, Game Theory (MIT Press, Cambridge, MA, 1993) 
30. S Lasaulce, H Tembine, Game Theory and Learning for Wireless Networks: Fundamentals and Applications (Elsevier, Amsterdam, 2011)

31. CU Saraydar, NB Mandayam, DJ Goodman, Efficient power control via pricing in wireless data networks. IEEE Trans. Commun. 50, 291-303 (2002)

32. E Altman, Z Altman, S-modular games and power control in wireless networks. IEEE Trans. Autom. Control 48, 839-842 (2003)

33. EE Tsiropoulou, P Vamvakas, G Katsinis, S Papavassiliou, Combined power and rate allocation in self- optimized multi-service two-tier femtocell networks. Elsevier Comput. Commun. J. 72, 38-48 (2015)

Submit your manuscript to a SpringerOpen ${ }^{\circ}$ journal and benefit from:

- Convenient online submission

Rigorous peer review

- Open access: articles freely available online

- High visibility within the field

- Retaining the copyright to your article

Submit your next manuscript at $\gg$ springeropen.com 